\title{
SUBSEMIGROUPS OF AMENABLE GROUPS
}

\section{HOCHSTER ${ }^{1}$}

Several authors have recently studied the problem of finding circumstances under which the amenability of a semigroup is inherited by a subsemigroup. It is known ([2] and [3]) that subgroups of an amenable group are amenable, but that in general subsemigroups of an amenable semigroup are not. We show here that subsemigroups of an amenbable group need not be amenable.

Frey proved in [4] that subsemigroups of a group inherit amenability if and only if the group contains no copy of the free semigroup $S$ on two generators ( $S$ is not amenable). The problem is discussed in detail in [5]. It is known [1] that a group generated by a copy of $S$ contained in it need not be free; the question is whether it can be far enough from free to be amenable. Since solvable groups are amenable [6], our claim that subsemigroups of amenable groups need not be amenable will be established if we can embed $S$ in a solvable group. We proceed to do this. Granirer conjectured in his review [MR $35 \# 3423$ ] of Wilde and Witz [7] that a group is amenable if and only if it contains no copy of $S$. It will follow that the "only if" part of this conjecture is quite false.

Let $B$ be the free additive abelian group on the family of generators $\{b(m, n)\}, m, n$ integers. Any permutation of these generators induces an automorphism of $B$. Let $x$ and $y$ be the automorphisms induced by $b(m, n) \mapsto b(m+1, n)$ and $b(m, n) \mapsto b(m, n+1)$, respectively. It is easy to see that the subgroup $C$ of Aut $B$ generated by $x$ and $y$ is the free abelian group on the generators $x, y$. We write $C$ additively, and we indicate the action of an element $c$ of $C$ on an element $b$ of $B$ by $b^{c}$. Let $G$ be the normal product of $B$ by $C$. $G$ consists of pairs $[c, b]$ and the multiplication is given by $\left[c_{1}, b_{1}\right][c, b]$ $=\left[c_{1}+c, b_{1}^{c}+b\right] . a[c, 0], b \mapsto[0, b]$ are embeddings of $C, B$ respectively into $G$, and $B$ is thus a normal abelian subgroup with quotient $G / B \cong C$. Hence, $G$ is solvable and in fact the second derived group $G^{\prime \prime}$ of $G$ is trivial. The same holds for every subgroup of $G$. Now, let $s=[x, b(0,0)]$ and $t=[y, b(0,0)]$.

Proposition. $s$ and $t$ are free generators for a copy of $S$.

Proof. Consider a monomial $U=u_{1} \cdots u_{d}$ of length $d \geqq 1$, where

Received by the editors June 7, 1968.

1 Research partially supported by NSF grant GP-8496. 
each $u_{i}$ is either $s$ or $t$. Suppose $s$ occurs $p$ times and $t$ occurs $q$ times. Then $p+q=d$, and $U$ is of the form $\left[p x+q y, \sum_{i=0}^{d-1} b\left(m_{i}, n_{i}\right)\right]$, where for each $i, m_{i} \geqq 0, n_{i} \geqq 0$, and $m_{i}+n_{i}=i$. (In particular, $\left(m_{0}, n_{0}\right)=(0,0)$ and $\left(m_{1}, n_{1}\right)=(1,0)$ or $(0,1)$ if $d \geqq 2$.) Moreover, if $d \geqq 2,\left(m_{1}, n_{1}\right)$ $=(1,0)$ or $(0,1)$, according as $u_{d}=s$ or $u_{d}=t$. These statements are proved by a trivial induction on $d$.

Now suppose that the distinct monomials $U=u_{1} \cdots u_{d}$ and $V=v_{1} \cdots v_{e}$ are equal. We must have that $d=e$ because the length can be recovered as the sum of coefficients of $x$ and $y$ in the first entry. Assume that $d$ is as small as possible. If $d \geqq 2$, the second entry will be a sum of $d-1$ terms, precisely one of which, $b\left(m_{1}, n_{1}\right)$, will have the property that the sum of its indices is 1 . It follows that $u_{d}=v_{d}$ (both are $s$ or both are $t$, according as $\left(m_{1}, n_{1}\right)=(1,0)$ or $(0,1))$. But then $u_{1} \cdots u_{d-1}=v_{1} \cdots v_{d-1}$, contradicting the choice of $d$. We can conclude that $d=1$, another contradiction since $s \neq t$, and the proposition is established.

COROLLARY. Let $F$ be the free group on two generators $f, g$ and let $F^{\prime \prime}$ be the second derived group of $F$. Then the images of $f$ and $g$ in $F / F^{\prime \prime}$ are free generators for a copy of $S$.

Proof. Let $\phi: F \rightarrow G$ be the unique homomorphism such that $\phi(f)=s$ and $\phi(g)=t$, and let $H=\phi(F)$. Then $\phi\left(F^{\prime \prime}\right)=H^{\prime \prime}$ is trivial, since $G^{\prime \prime}$ is, and since $H \cong F / \operatorname{Ker} \phi$, we must have $F^{\prime \prime} \subset \operatorname{Ker} \phi$. Since the images of $f$ and $g$ are free generators for a copy of $S$ in $F / \operatorname{Ker} \phi$, this certainly holds in $F / F^{\prime \prime}$ as well.

This embedding of $S$ into $F / F^{\prime \prime}$ is a "generic" counter example.

I wish to thank G. Keller and C. Wilde for pointing this problem out to me and supplying helpful background.

\section{REFERENCES}

1. K. Appel and F. Djorup, On the group generated by a free semigroup, Proc. Amer. Math. Soc. 15 (1964), 838-841.

2. M. M. Day, Amenable semigroups, Illinois J. Math. 1 (1957), 509-544.

3. E. Følner, Note on groups with and without full Banach mean value, Math. Scand. 5 (1957), 5-11.

4. A. H. Frey, Jr., Studies in amenable semigroups, Thesis, University of Washington, Seattle, Washington, 1960.

5. E. Granirer, Extremely amenable semigroups. II, Math. Scand. 20 (1967), 93-133.

6. J. von Neumann, Zur allgemeinen Theorie des Masses, Fund. Math. 13 (1929), 73-116.

7. C. Wilde and K. Witz, Invariant means and the Stone-Čch compactification, Pacific J. Math. 21 (1967), 577-586.

UNiversity of MinNesota 